\title{
Cost-effectiveness of the HiBalance training program for elderly with Parkinson's disease: analysis of data from a randomized controlled trial
}

Conran Joseph, Nina Brodin, Breiffni Leavy, Maria Hagströmer, Niklas Löfgren and Erika Franzén

\begin{abstract}
Objective: To determine the cost-effectiveness of the HiBalance training program for managing Parkinson's disease (PD)-related balance and gait disorders.

Design: Cost comparison design following the randomized controlled trial comparing a novel balance training intervention with care as usual.

Subjects: A total of 100 participants with mild-moderate PD were randomized to either the intervention $(n=51)$ or the control group $(n=49)$.

Intervention: A 10-week (three times per week), group-based, progressive balance training program, led by two physical therapists.

Main outcomes: All program costs were collected for both groups. Cost-utility was evaluated using quality-adjusted life years (QALYs) and cost-effectiveness measures were the Mini Balance Evaluation Systems Test (Mini-BESTest; assessing balance performance) and gait velocity. Incremental cost-effectiveness ratios were calculated and a probabilistic sensitivity analysis was conducted.

Results: The between-group difference in QALYs was 0.043 (95\% confidence interval (CI): 0.011-0.075), favoring the intervention group. Between-group differences in balance performance and gait velocity were 2.16 points (95\% CI: $1.19-3.13$ ) and $8.2 \mathrm{~cm} / \mathrm{second}$ (95\% CI: 2.9-13.6), respectively, favoring the intervention group. The mean cost per participant in the intervention group was 16,222 SEK (€1649) compared to 2696 SEK (€274) for controls. The estimated incremental cost-effectiveness ratios were 314,558 SEK $(€ 31,969)$ for an additional QALY, 6262 SEK (€631) for one point improvement in balance performance, and 1650 SEK (€166) for $1 \mathrm{~cm} /$ second increase in gait velocity. Sensitivity analyses indicated a high probability (85\%) of program success.

Conclusion: In terms of QALYs, the HiBalance program demonstrated a high probability of cost-effectiveness in the short-term perspective when considering the willingness-to-pay thresholds used in Europe.
\end{abstract}

\section{Introduction}

Impaired balance is a major issue in people with Parkinson's disease (PD). The lack of sufficient balance performance predisposes people with PD to potentially injurious falls 
which, in turn, compromise one's quality of life, livelihood, and functioning. ${ }^{1-3}$ A specific training regime, the HiBalance training program consisting of highly challenging dynamic balance exercises, has been developed in Sweden.4,5 The program was found to be effective in improving balance and gait velocity, which exceeded the minimal clinically important difference of $0.05 \mathrm{~m} / \mathrm{second}$ (for gait velocity), as commonly used in elderly and persons with PD.6,7 Given the promising findings in the earlier randomized controlled trial, 8 the need exists to evaluate the cost-effectiveness of the HiBalance program for consideration by health-care decision-makers who are responsible for strengthening healthcare systems, especially in rehabilitation.

In an era with limited resources for health services, it is important to evaluate the economic aspects of interventions, with the aim of selecting the option associated with the best value. The HiBalance program was developed in a time when a distinct need for rehabilitation interventions targeting motor symptoms existed, and was found to be effective as well as highly appreciated by participants. 8,9 With the next step directed toward the implementation of the program, its cost-effectiveness needs to be established. Therefore, the aim of this cost comparison study was to determine the cost-utility, accounting for costeffectiveness, of the HiBalance training program in terms of changes in health-related quality of life as well as balance performance and gait velocity over a 10-week intervention period.

\section{Methods}

\section{Design and participants}

An economic evaluation of the HiBalance program was carried out whereby costs and the associated outcomes of the HiBalance program versus regular care were compared. The effects of the HiBalance program were previously examined in a single-blinded, randomized, controlled trial which recruited participants between January 2012 and July 2013. 8 In total, 146 participants were recruited through advertisements in local newspapers, Karolinska University Hospital, and outpatient neurological clinics in Stockholm County, Sweden. The Regional Ethical Board in Stockholm provided ethical clearance (dnr: 2009/819-32, 2010/1472-32, 2012/1829-32), and the study was subsequently registered in ClinicalTrials.gov (trial registration no. NCTo1417598).

To be eligible for participation, clients had to present with a clinical diagnosis of idiopathic PD, have a Hoehn and Yahr ${ }^{10}$ score of 2 or 3, be 60 years or above, independently ambulate indoors without a walking aid, and be on a stable dose of anti-Parkinson's medication for at least three weeks. The exclusion criteria were as follows: (1) impaired mental function which was confirmed by a score less than 24 on the Mini-Mental State Examination ${ }^{11}$ and (2) the presence of other medical conditions which may influence the main study outcomes. 


\section{Interventions}

The HiBalance training program was developed to specifically target balance impairments in persons with PD, relating to the following four components: (1) sensory integration, (2) anticipatory postural adjustments, (3) motor agility, and (4) stability limits. The 10-week (three times per week) program was group-based, consisted of 4-7 participants, and facilitated by two trained physiotherapists at all sessions. Each session lasted 6o minutes and consisted of a 5-minute warm-up, 50-minute highly challenging balance exercises, and a 5 -minute cool-down period at the end. The training program contained exercises that were individualized to the ability of participants. Importantly, no fixed regime of predetermined exercises was used; instead, motor learning principles, including specificity, progressive overload, and variation, were adopted for the application and adaptation of exercises to suit participants' abilities. To address specificity, exercises were linked to the four balance components, and variation was achieved by integrating and combining different balance components within a single session or activity as the program progressed through the three distinct stages (also called Blocks A, B, and C). In addition, dual-tasking, either cognitive (e.g. counting and remembering items) or motor (e.g. carrying or manipulating objects), was gradually integrated to aid progression. Progressive overload was further enhanced by increasing the complexity of exercises once participants made fewer to no errors by, for example, increasing surface unevenness to challenge sensory integration and increasing movement amplitude and velocity to challenge anticipatory postural adjustments. Further details of the HiBalance program can be found in previous publications. $4,5,8$

Participants randomized into the control group received usual care from their healthcare service providers. Participants in the control group were allowed to enroll in and/or continue with their organized health-enhancing activities, such as training by oneself or participating in exercise classes. All participants serving as controls were offered the HiBalance program at the end of the study period.

\section{Outcomes and costs}

Participants, in both the intervention and control groups, were evaluated at baseline and after the intervention which lasted 10 weeks. Cost-utility was assessed in terms of gains in quality-adjusted life years (QALYs). The Short Form-36 version 1 was used to evaluate generic quality of life. The Short Form-36 raw data were transformed to a Short Form6D utility index score. The Short Form-6D utility scores come with a set of preference weights obtained from a sample of the general population in the United Kingdom, using the standard gamble valuation technique. 12 Values were given by the reference population to a selection of health states from which a model had been developed to predict all the health states described by the Short Form-6D.13 The score ranges from zero to one, with zero being dead and closer to one suggesting "almost perfect health."

Cost-effectiveness outcomes were balance performance and gait velocity. Balance performance was assessed using the Mini Balance Evaluation Systems Test (Mini- 
BESTest). It assesses the underlying subsystems of balance performance (anticipatory postural adjustment, postural responses, sensory integration, and dynamic gait) on a threelevel ordinal scale. The total score ranges from o to 28 , with a higher score reflecting better balance performance.14 Gait velocity was assessed using a 9-meter electronic walkway (GAITRite; CIR Systems Inc., Clifton, NJ, USA) and expressed in cm/second.

A cost comparison approach was implemented to study the cost-effectiveness of the training program versus care as usual. Although not exhaustive, direct and indirect, as well as social, costs were considered and formed part of the estimation of costs for each intervention. Intervention-related costs were retrospectively collected, and for that reason an annual inflation rate of $1 \%$ was added to present what the HiBalance intervention would cost today. Inflation in Sweden has remained low over the last five years, with a year-onyear rate ranging from -0.31 to 1.74 (http://www.inflation.eu/inflation-rates/cpiinflation.aspx).

Costs related to both groups included (1) education of physiotherapists for assessments, (2) participants' and therapists' costs during assessments, and (3) equipment and facilities used during assessments. Additional intervention-related costs included 30 hours of HiBalance training per person, that is, trainers' salary costs, delivered three times weekly for 10 weeks, costs for hiring the venue, and participants' time costs (3o hours). An additional 30 hours (1 hour per session) was added to the trainers' costs for preparation and planning, an important aspect of the program for ensuring individualized and progressive balance exercises, and documentation following the training session. Furthermore, an additional 30 hours was added to participants' time to account for traveling to and from training venue.

Since cost data were retrospectively collected, we were unable to precisely estimate trainers' and participants' time costs. Our estimate therefore represents the mean hourly wage of physiotherapists who delivered the education and training (2012/2013) in this study. Participants' costs were similarly estimated by adopting the mean hourly wage of retired people in Sweden during the year 2012/2013. It is important to note that the total cost estimate presented in this study does not include costs incurred by additional healthcare (such as medications) and other health-enhancing leisure activities.

\section{Statistical analysis}

Data were analyzed in Stata (STATA Corp, College Station, TX, USA) version 13, where an intention-to-treat approach was followed. For the description of baseline data between groups, the chi-square test was used for nominal data and independent-samples $t$-test for continuous data. Levene's test, in combination with visual inspection, was used to determine the normality of outcomes used in this study. Since all outcomes fulfilled these criteria, multivariate linear regression analysis was used to estimate the mean $(95 \%$ confidence interval (CI)) between-group differences for QALYs, balance performance, and gait speed by controlling for baseline utility/score, group allocation, and disease severity. 
Cost-effectiveness was calculated by considering the intervention costs per participant in relation to the net effect seen in the Short Form-6D utility score, balance performance, and gait velocity. The comparator was "usual care" as previously described. Incremental


control)) were calculated to determine the costs associated with every unit increase in the selected study outcomes (see Table 4). A probabilistic sensitivity analysis was performed, with cost-effectiveness acceptability curves produced, to test for uncertainty around incremental cost-effectiveness ratios. The effectiveness and utility estimations have been replicated on 10,000 non-parametric bootstrap samples, stratified by treatment group. Based on the set of 10,000 estimates from the bootstrap procedure, cost-effectiveness acceptability curves have been produced against a range of values representing society's willingness to pay for such health-enhancing interventions. The cost-effectiveness analysis was performed using R (RStudio v 1.1.419). The boot function was used for the bootstrap sampling, and the icea function of the hesim package was used to produce results for the cost-effectiveness acceptability curves.

\section{Results}

\section{Participants' characteristics}

A total of 100 participants were enrolled in this study, with 51 and 49 allocated to the intervention and control groups, respectively (Figure 1). As shown in Table 1, no significant differences were present between the groups with respect to demographics, anthropometric measures, clinical features, or baseline utility and effectiveness outcomes.

\section{Effects of the intervention}

Concerning the utility outcome, a significant difference, according to the transformed Short Form-6D utility score, was noted in the intervention group following the treatment period but not in the control group (Table 2). The estimated between-group difference in utility for the period under study was 0.043 QALYs (95\% CI: 0.011-0.075), benefiting the intervention group. Furthermore, a significant improvement of 2.16 points (95\% CI: 1.18-3.12) was observed for balance performance in the intervention group, compared with controls, following the HiBalance training. 




Figure I. Consort flowchart illustrating participant recruitment, retention, and loss to follow-up.

Similarly, a significant improvement of $8.2 \mathrm{~cm} /$ second (95\% CI: $3.0-13.6$ ) in gait velocity was observed when comparing the mean difference estimate between groups, again favoring the intervention group.

\section{Intervention costs}

The total cost for the intervention was 959,413 SEK (€97,535), with a larger proportion of the spending directed toward the intervention group (86\%). 
Table I. Participants' baseline characteristics.

\begin{tabular}{lccc}
\hline & Intervention group $(N=51)$ & Control group $(N=49)$ & $P$ \\
\hline Gender, male/female & $32 / 19$ & $25 / 24$ & 0.24 \\
Mean age (years)/SD & $73.1 / 5.8$ & $73.0 / 5.5$ & 0.92 \\
Body weight $(\mathrm{kg})$, mean/SD & $75.7 / 13.6$ & $76.0 / 14.0$ & 0.94 \\
Body height, mean/SD & $172.2 / 8.9$ & $171.2 / 9.0$ & 0.59 \\
H\&Y stage, $2 / 3$ & $23 / 28$ & $21 / 28$ & 0.82 \\
UPDRS motor & $37.5 / 10.9$ & $35.7 / 10.0$ & 0.39 \\
PD duration (years), mean/SD & $5.9 / 5.1$ & $5.6 / 4.8$ & 0.78 \\
LED medication, mean/SD & $569 / 283$ & $652 / 397$ & 0.23 \\
Mini-BESTest (0-28), mean/SD & $19.1 / 3.1$ & $18.4 / 3.5$ & 0.25 \\
Normal gait velocity, mean/SD & $1.29 / .26$ & $1.30 / .22$ & 0.87 \\
SF-6D utility score, mean/SD & $0.64 / 0.11$ & $0.66 / 0.12$ & 0.59 \\
\hline
\end{tabular}

H\&Y: Hoehn and Yarn; UPDRS: Unified Parkinson's Disease Rating Scale; PD: Parkinson's disease; LED: Levodopa Equivalency Dosage; Mini-BESTest: Mini Balance Evaluation Systems Test; SF-6D: Short Form-6 Dimension.

Table 2. Pre-post intervention scores for utility and effectiveness measures.

\begin{tabular}{lcclll}
\hline Measures & \multicolumn{2}{l}{ Intervention group mean $(95 \% \mathrm{Cl})$} & & \multicolumn{2}{l}{ Control group mean $(95 \% \mathrm{Cl})$} \\
\cline { 2 - 3 } \cline { 5 - 6 } & Pre & Post & & Pre & Post \\
\hline SF-6D utility score $(0-1)$ & $0.65(0.62-0.68)$ & $0.70(0.67-0.73)$ & & $0.66(0.63-0.69)$ & $0.67(0.64-0.70)$ \\
Mini-BESTest $(0-28)$ & $19.18(18.29-20.06)$ & $21.96(21.11-22.81)$ & & $18.33(17.33-19.32)$ & $19.14(18.01-20.28)$ \\
Normal gait velocity & $1.19(1.13-1.25)$ & $1.27(1.22-1.32)$ & & $1.15(1.10-1.20)$ & $1.16(1.11-1.22)$ \\
\hline
\end{tabular}

Cl: confidence interval; SF-6D: Short Form-6 Dimension; Mini-BESTest: Mini Balance Evaluation Systems Test.

As shown in Table 3, the estimated mean difference in total costs between the intervention and control groups was 13,526 SEK ( $€ 1375)$ per participant.

\section{Cost-effectiveness analysis of the intervention}

As shown in Table 4, the incremental cost associated with an improvement in 1 QALY is estimated at 314,558 SEK $\left(€_{31}, 712\right)$. The incremental cost-effectiveness ratio for balance performance was 6262 SEK (€631; one unit increase in the Mini-BESTest score per patient in the intervention group). Gait velocity improved by approximately $8 \mathrm{~cm}$ in the intervention group, resulting in a corresponding incremental cost-effectiveness ratio of 1650 SEK (€166) per unit (i.e. $1 \mathrm{~cm}$ ).

\section{Uncertainty analysis}

The cost-effectiveness acceptability curves (Figures 2-4) show the robustness of the model regarding the uncertainty estimation of the outcomes for each group. These costeffectiveness acceptability curves included the association between the values of the ceiling ratio (e.g. willingness to pay for a unit of QALY gained) and the probability of favoring the intervention group. Concerning the uncertainty around the QALY outcome, the results showed that the HiBalance intervention has an $85 \%$ probability of being costeffective given a willingness to pay of 500,000 SEK per QALY. The results concerning balance 
performance indicate that the intervention has $70 \%$ and $85 \%$ probability of producing a sufficiently large treatment effect if the payer is willing to pay 7000 and 8000 SEK, respectively, for one unit increase. Furthermore, the intervention has $72 \%$ and $86 \%$ probability of producing a sufficiently large treatment effect if the payer is willing to pay 2000 and 2500 SEK, respectively, for every centimeter/second improvement in gait velocity.

Table 3. Program costs, both direct and indirect costs for the intervention and control groups.

\begin{tabular}{|c|c|c|c|c|c|}
\hline \multirow[t]{2}{*}{ Costs in SEK } & \multicolumn{2}{|c|}{ Intervention group $(n=51)$} & \multicolumn{2}{|c|}{ Control group $(n=49)$} & \multirow[t]{2}{*}{$\Delta$ per patient } \\
\hline & Total cost & Per Patient & Total cost & Per patient & \\
\hline \multicolumn{6}{|l|}{ Direct costs } \\
\hline \multicolumn{6}{|l|}{ Training education (6 hours) } \\
\hline Renting of venues & 1300 & & & & \\
\hline Salary of teacher & 2378 & & & & \\
\hline Salary of assistant teacher & 2605 & & & & \\
\hline Total training costs & 6283 & 123 & & & \\
\hline \multicolumn{6}{|l|}{ Equipment and training } \\
\hline Balance training equipment & 24,421 & & & & \\
\hline Training hall for 10 groups & 90,000 & & & & \\
\hline Trainers' salary & 260,400 & & & & \\
\hline Equipment and training costs & 374,821 & 7349 & & & \\
\hline \multicolumn{6}{|l|}{ Assessment costs } \\
\hline Testing equipment & 1175 & & 1175 & & \\
\hline Training of assessors & 4330 & & 4330 & & \\
\hline Assessment time (assessors) & 43,400 & & 43,400 & & \\
\hline Testing venue & 52,500 & & 52,500 & & \\
\hline Total assessment costs & 101,405 & 1988 & 101,405 & 2070 & \\
\hline \multicolumn{6}{|l|}{ Indirect costs } \\
\hline Participants' time (assess)a & 30,600 & & 29,400 & & \\
\hline Participants' (IG) time Rx & 306,000 & & & & \\
\hline Total indirect costs & 336,600 & & 29,400 & & \\
\hline Total costs SEK (societal) & 819,109 & 16,061 & 130,805 & 2670 & $|3,39|$ \\
\hline Total cost plus $1 \%$ inflation & 827,300 & 16,222 & 132,113 & 2696 & 13,526 \\
\hline Total cost in $€$ & 83,393 & 1635 & 13,317 & 272 & 1363 \\
\hline
\end{tabular}

IG: intervention group.

'Participants' time during assessment totaled 5 hours, with 4 hours reflecting pre and post assessment $(2+2)$ and I hour traveling to and from the testing venue.

bParticipants' time during treatment totaled 60 hours over the 10 -week training program, 30 hours for the actual training (three times weekly for 10 weeks) and 30 hours for traveling to and from the treatment venue $(3 \times 10)$. 
Table 4. Incremental cost-effectiveness ratios in SEK for study outcomes.

\begin{tabular}{llrr}
\hline Outcome measures & \multicolumn{2}{l}{ Total population } & ICER (SEK) \\
\cline { 2 - 3 } & $\Delta$ effect & $\Delta$ cost & \\
\hline SF-6D & 0.043 & 13,526 & 314,558 \\
Mini-BESTest $(0-28)$ & 2.16 & 13,526 & 6262 \\
Gait velocity $(\mathrm{cm} /$ second) & 8.2 & 13,526 & 1650 \\
\hline
\end{tabular}

ICER: incremental cost-effectiveness ratio; SF-6D: Short Form-6 Dimension; Mini-BESTest: Mini Balance Evaluation Systems Test.

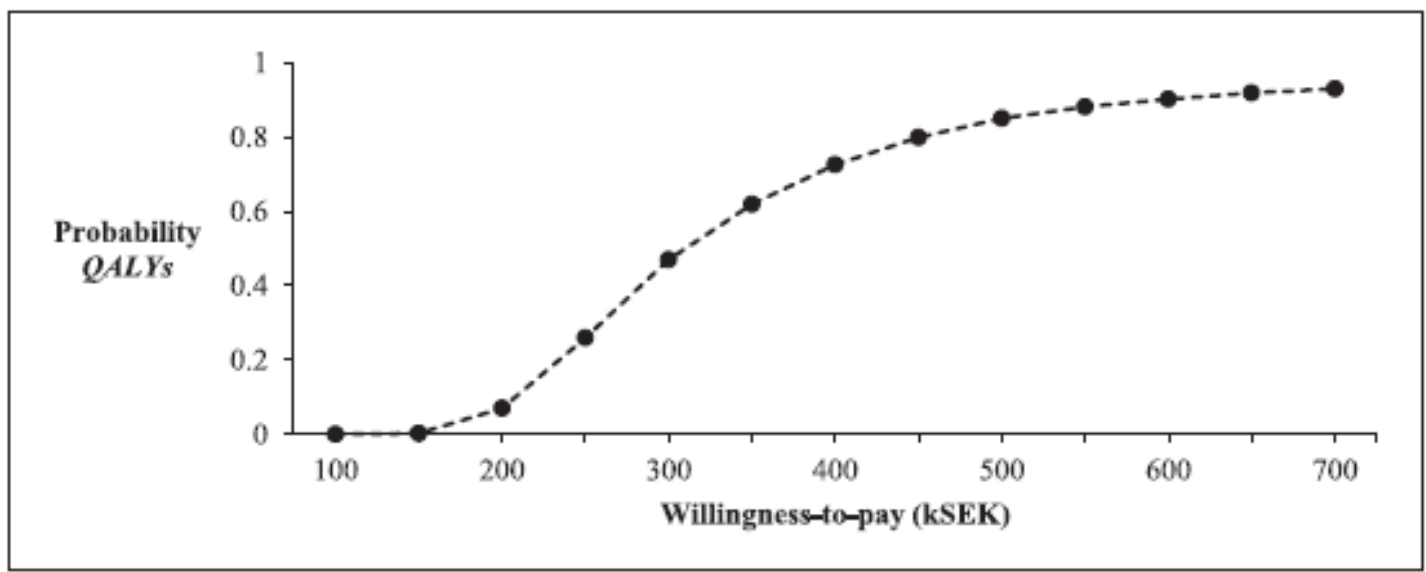

Figure 2. Cost-effectiveness acceptability curve for the QALY outcome.

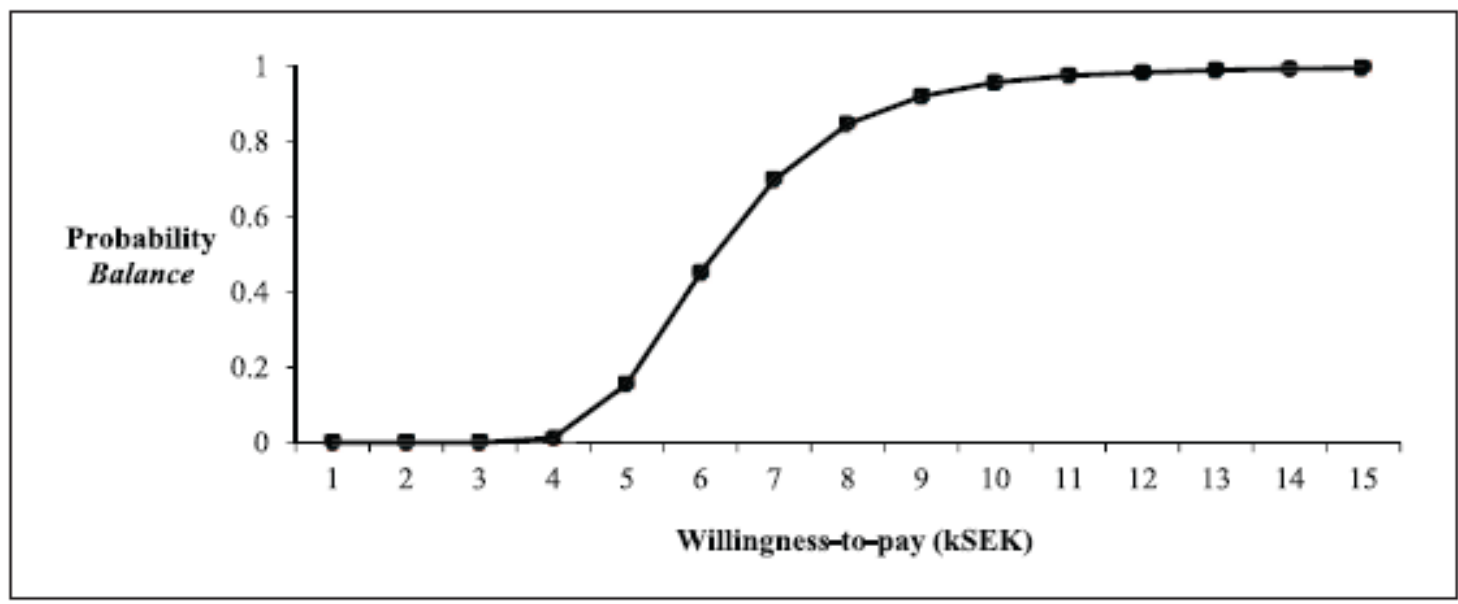

Figure 3. Cost-effectiveness acceptability curve for the balance outcome. 


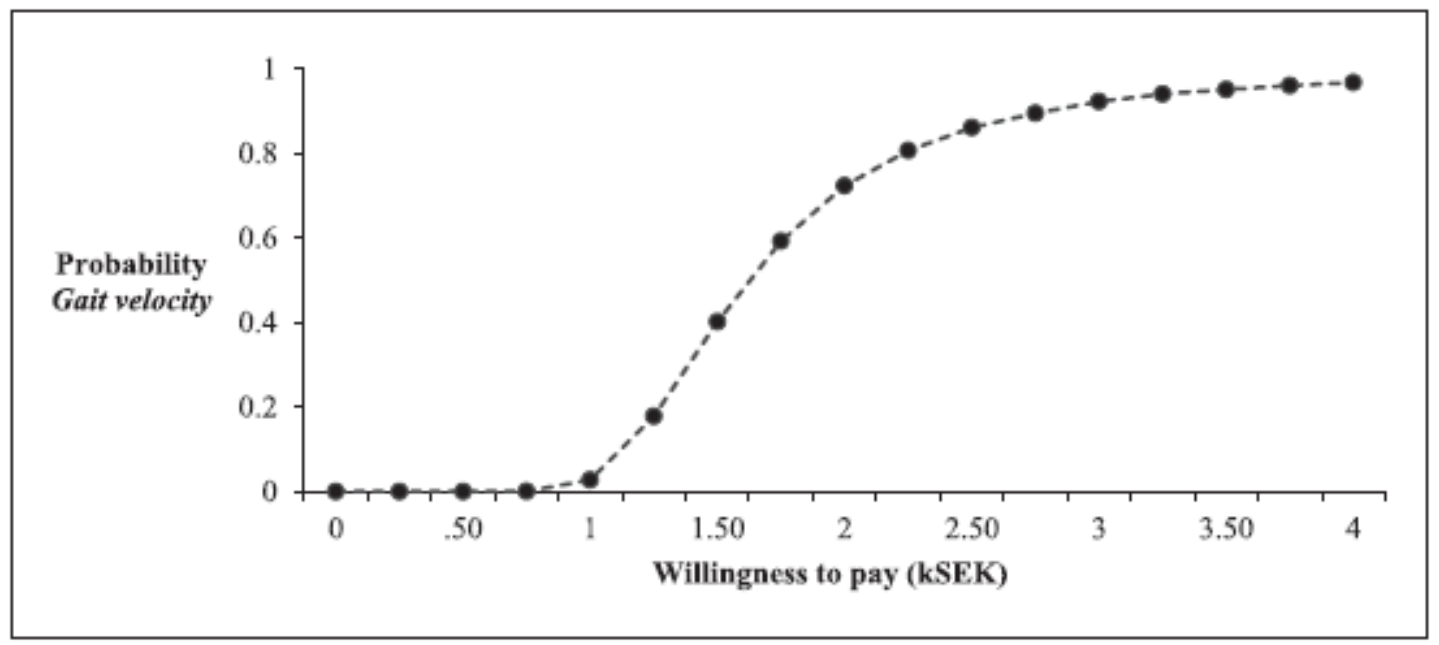

Figure 4. Cost-effectiveness acceptability curve for the gait velocity outcome.

\section{Discussion}

This study assessed the cost-utility, as well as cost-effectiveness, of a novel balance training program for persons with mild-moderate PD enrolled in a randomized controlled trial. The analysis revealed that the HiBalance intervention is good value for money, according to gains made in QALYs, in the short-term perspective. It further shows that improvements in balance performance and gait velocity are achievable at a reasonable cost, albeit more expensive than usual care.

Adecision on an intervention's cost-effectiveness depends upon gains made in QALYs, and the willingness of healthcare decision makers/society to pay for them. We found a significant difference in QALY between groups, favoring those receiving the intervention. In absolute terms, this 10-week program added, on average, an additional 15 days of perfect health to


best-evidence approaches, no formal monetary value for a QALY exists in Sweden. However, a published review of the Swedish government agency responsible for deciding whether drug interventions are covered by its scheme found a value of $1,220,000$ SEK to be the highest cost per QALY approved for reimbursements. 15 It further found that at a cost per QALY of 702,000 SEK for non-severe diseases and 988,000 SEK for severe diseases, the likelihood of approval was estimated to be 50/50.

The preservation of functioning in persons with PD remains a top priority. To realize this, physiotherapy as well as occupational therapy is an essential requirement. ${ }^{16}$ As highlighted by Tomlinson et al.,7 very few studies in the rehabilitation arena incorporate economic evaluations alongside pragmatic randomized controlled trials, which, in turn, hampers the identification of cost-effective interventions targeting disabling symptoms. Although not similar in intervention, one cost-effectiveness study investigating a physical activity intervention among elderly found similar effects on QALY as in this study, albeit slightly more costly. 17 It should be noted that our study included fewer costs, which may have resulted 
in the different thresholds reached in these studies. Another systematic review also reported overall cost-effectiveness of brief physical activity interventions on QALY,18 while exercise and other physiotherapy-related modalities were also found to be costeffective in the management of osteoarthritis, considering a willingness-to-pay threshold of approximately $€_{50,000 .}{ }^{19}$ Specific to this study, we found that the HiBalance intervention had a high probability (85\%) of being cost-effective against a willingness-to-pay threshold of 500,000 SEK (€50,000).

As observed in this study, disease-relevant outcomes improved significantly over the course of the intervention period. First, balance performance increased, on average, by two units according to the Mini-BESTest. Although the mean estimate change in balance performance did not meet the threshold for minimal clinically important difference, 20 it should however be considered in conjunction with gait velocity which improved beyond its minimally clinically important difference of $5 \mathrm{~cm} / \mathrm{second}$ often used in PD.7 This balance training program offered similar effects on balance performance and gait velocity as other comparable physiotherapy interventions previously investigated in randomized controlled trials.21,22 Maintenance and improvement of gait velocity has been established as an important target among elderly populations, mainly due to its association with compromised quality-of-life states 23 and mortality, specifically that of cardiovascular origin.24,25 To our knowledge, no published studies investigated the benefits of balance training in relation to its associated costs. Our reported costs per unit increase in balance performance and gait velocity demonstrate how significant changes in health outcomes represent only a small fraction of annual patient healthcare costs. ${ }^{26}$ Sensitivity analysis of this program's cost-effectiveness revealed a high probability of success albeit at a higher cost than usual care, where the latter typically includes no structured programs. It is however difficult to interpret the willingness-to-pay thresholds for balance and gait velocity improvements in our study, since no other published health economic evaluations in the Parkinson's rehabilitation field are available to serve as a comparator.

Not all interventions are equally effective, let alone cost-effective. A range of studies suggest the use of intensive and challenging exercises in promoting and maintaining functioning, specifically gait speed and motor strength, above usual care.27,28 The HiBalance training program under investigation in this study is one of the few effective physiotherapy interventions directed at targeting functional decline among persons with PD. It should be considered for wider adoption considering its clear theoretical underpinnings, safety, and newly established cost-effectiveness. Such information is required for successful uptake of interventions in practice.29

This study presents with several limitations which require consideration. The retrospective approach to cost estimation prevented the actualization of exact costs of some expenses, for example, participants'time and travel costs. Furthermore, certain costs, specifically related to medication and additional healthcare/hospitalizations, as well as sick leave benefits, were 
omitted since the necessary permissions were not in place. These omissions have, most likely, affected total cost estimates and the cost per utility, which might influence the decision making around society's willingness to pay for this intervention. Furthermore, the use of United Kingdom's preference-based weights for deriving at the Short Form-6D utility scores for our cohort can be seen as another limitation. There is a risk that Swedes may hold different health state preferences than citizens from the United Kingdom. Finally, an obvious limitation of the sensitivity analysis was that intervention costs were fixed for the two groups due to the research paradigm being a controlled trial. To promote wider access of this effective rehabilitation intervention, future studies should use Swedish tariffs for utility measures and include all related healthcare costs during and after the intervention in order to convince healthcare decision-makers about the necessity of these health-enhancing interventions.

Overall, the results demonstrated that the HiBalance intervention is likely to induce better health states as well as balance performance and gait speed than usual care but at a higher cost. This intervention appears promising in terms of both delivering desired effects and its cost. However, prior to the wider implementation of the HiBalance training program, a more comprehensive assessment of costs, from a full societal perspective, along with a longer follow-up period of costs and outcomes collection, should be carried out in order to affirm its cost-utility and cost-effectiveness.

\section{Clinical Messages}

- The HiBalance training program was more costly than usual care but also delivered significantly better effects on utility and effectiveness outcomes.

- The HiBalance training program demonstrated a high probability of being cost-effective at a willingness-to-pay threshold of $500,000 \mathrm{SEK}(€ 50,000)$. 


\section{Acknowledgements}

We would like to thank all people with PD who have participated in this study.

\section{Declaration of Conflicting Interests}

The author(s) declared no potential conflicts of interest with respect to the research, authorship, and/or publication of this article.

\section{Funding}

The author(s) disclosed receipt of the following financial support for the research, authorship, and/or publication of this article: We are grateful for the financial support from the Swedish Research Council (2016-01965), the Swedish Research council for Health, Working life and Welfare (FORTE; 2014-4764), and "Vårdalstiftelsen" (2014-79). These funding agencies had no role in the design, collection, and interpretation of data and writing of the manuscript.

\section{ORCID iDs}

Conran Joseph https://orcid.org/oooo-0002-3121-2685

Erika Franzén https://orcid.org/oooo-0oo1-5520-544X 


\section{References}

1. Kerr GK, Worringham CJ, Cole $\mathrm{MH}$, et al. Predictors of future falls in Parkinson disease. Neurology 2010; 75(2): 116-124.

2. Kim SD, Allen NE, Canning CG, et al. Postural instability in patients with Parkinson's disease. Epidemiology, pathophysiology and management. CNS Drugs 2013; 27(2): 97-112.

3. Pickering RM, Grimbergen YA, Rigney U, et al. A meta-analysis of six prospective studies of falling in Parkinson's disease. Mov Disord 2007; 22(13): 1892-1900.

4. Conradsson D, Lofgren N, Stahle A, et al. A novel conceptual framework for balance training in Parkinson's disease-study protocol for a randomised controlled trial. BMC Neurol 2012; 12(111): 1471-2377.

5. Conradsson D, Lofgren N, Stahle A, et al. Is highly challenging and progressive balance training feasible in older adults with Parkinson's disease? Arch Phys Med Rehabil 2014; 95(5): 1000-1003.

6. Perera S, Mody SH, Woodman RC, et al. Meaningful change and responsiveness in common physical performance measures in older adults. $J$ Am Geriatr Soc 2006; 54(5): 743-749.

7. Tomlinson CL, Patel S, Meek C, et al. Physiotherapy intervention in Parkinson's disease: systematic review and meta-analysis. BMJ Brit Med $J$ 2012; 345: e5004.

8. Conradsson D, Lofgren $\mathrm{N}$, Nero $\mathrm{H}$, et al. The effects of highly challenging balance training in elderly with Parkinson's disease: a randomized controlled trial. Neurorehabil Neural Repair 2015; 29(9): 827-836.

9. Leavy B, Roaldsen KS, Nylund K, et al. "Pushing the limits": rethinking motor and cognitive resources after a highly challenging balance training program for Parkinson disease. Phys Ther 2017; 97: 81-89.

10. Hoehn MM and Yahr MD. Parkinsonism: onset, progression and mortality. Neurology 1967; 17(5): 427-442.

11. Folstein MF, Folstein SE and McHugh PR. "Mini-mental state." A practical method for grading the cognitive state of patients for the clinician. $J$ Psychiatr Res 1975; 12(3): 189-198.

12. Brazier J, Usherwood T, Harper R, et al. Deriving a preference-based single index from the UK SF-36 Health Survey. J Clin Epidemiol 1998; 51(11): 1115-1128.

13. Brazier JE and Roberts J. The estimation of a preference-based measure of health from the SF-12. Med Care 2004; 42(9): 851-859.

14. Franchignoni F, Horak F, Godi M, et al. Using psychometric techniques to improve the Balance Evaluation Systems Test: the mini-BESTest. J Rehabil Med 2010; 42(4): 323-331.

15. Svensson M, Nilsson FO and Arnberg K. Reimbursement decisions for pharmaceuticals in Sweden: the impact of disease severity and cost effectiveness. Pharmacoeconomics 2015; 33(11): 1229-1236.

16. Fox SH, Katzenschlager R, Lim SY, et al. The Movement Disorder Society evidencebased medicine review update: treatments for the motor symptoms of Parkinson's disease. Mov Disord 2011; 26(suppl. 3): S2-S41. 
17. Groessl EJ, Kaplan RM, Castro Sweet CM, et al. Cost-effectiveness of the LIFE physical activity intervention for older adults at increased risk for mobility disability. J Gerontol A Biol Sci Med Sci 2016; 71(5): 656-662.

18. GC V, Wilson EC, Suhrcke M, et al. Are brief interventions to increase physical activity cost-effective? A systematic review. Br J Sports Med 2016; 50: 408-417.

19. Pinto D, Robertson MC, Hansen P, et al. Cost-effectiveness of nonpharmacologic, nonsurgical interventions for hip and/or knee osteoarthritis: systematic review. Value Health 2012; 15(1): 1-12.

20.Godi M, Franchignoni F, Caligari M, et al. Comparison of reliability, validity, and responsiveness of the mini-BESTest and Berg Balance Scale in patients with balance disorders. Phys Ther 2013; 93(2): 158-167.

21. Tomlinson CL, Herd CP, Clarke CE, et al. Physiotherapy for Parkinson's disease: a comparison of techniques. Cochrane Database Syst Rev 2014; 6: CDoo2815.

22. Sparrow D, DeAngelis TR, Hendron $K$, et al. Highly challenging balance program reduces fall rate in Parkinson disease. $J$ Neurol Phys Ther 2016; 40(1): 24-30.

23. Herman T, Giladi N, Gruendlinger L, et al. Six weeks of intensive treadmill training improves gait and quality of life in patients with Parkinson's disease: a pilot study. Arch Phys Med Rehabil 2007; 88(9): 1154-1158.

24. Dumurgier J, Elbaz A, Ducimetière P, et al. Slow walking speed and cardiovascular death in well functioning older adults: prospective cohort study. $B M J$ 2009; 339: b4460.

25. Studenski S, Perera S, Patel K, et al. Gait speed and survival in older adults. JAMA 2011; 305(1): 50-58.

26. Kowal SL, Dall TM, Chakrabarti R, et al. The current and projected economic burden of Parkinson's disease in the United States. Mov Disord 2013; 28(3): 311-318.

27. Shulman LM, Katzel LI, Ivey FM, et al. Randomized clinical trial of 3 types of physical exercise for patients with Parkinson disease. JAMA Neurol 2013; 70(2): 183-190.

28. Frazzitta G, Balbi P, Maestri R, et al. The beneficial role of intensive exercise on Parkinson disease progression. Am J Phys Med Rehabil 2013; 92(6): 523-532.

29. Allen NE, Sherrington C, Suriyarachchi GD, et al. Exercise and motor training in people with Parkinson's disease: a systematic review of participant characteristics, intervention delivery, retention rates, adherence, and adverse events in clinical trials. Parkinson's Dis 2012; 2012: 854328. 